\title{
$R$. rattus y $R$. norvegicus, como reservorio de endoparásitos zoonóticos en Ecuador
}

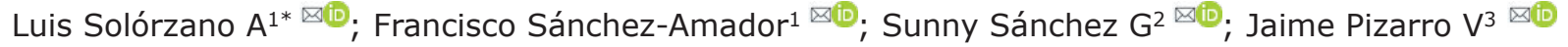

\begin{abstract}
${ }^{1}$ Centro de Referencia Nacional de Parasitología. Instituto Nacional de Investigación en Salud Pública "Leopoldo Izquieta Pérez" de Guayaquil. Ecuador.

2Universidad Especialidades Espíritu Santo, Escuela de Medicina. Av. Samborondón, Samborondón. Ecuador.

3Universidad Estatal de Guayaquil, School of Medicine. Cdla. Universitaria Salvador Allende Malecón del Salado. Ecuador.

*Correspondencia: luisfernando.solorzano@gmail.com
\end{abstract}

Recibido: Marzo 2020; Aceptado: Diciembre 2020; Publicado: May 2021.

\section{RESUMEN}

Objetivo. Recolectar información sobre la presencia de parasitosis intestinales en roedores, con énfasis en parásitos de importancia médica en humanos. Materiales y métodos. Se realizó un estudio parasitológico para detectar la presencia de helmintos en roedores en zonas urbanas y rurales de cinco provincias del Ecuador entre el 2014 y 2017. Los roedores fueron capturados y transportados al Centro Referencia Nacional de Parasitología del Instituto Nacional de Investigación en Salud Pública - Guayaquil, para su posterior análisis. Resultados. Se encontraron 125/211 (59.2\%) ratas con endoparásitos, $13 / 20(65 \%) R$. rattus y 112/191 (58.6\%) $R$. norvegicus. El nemátodo más prevalente fue Nippostrongylus brasiliensis (para ambas especies de roedores), y Heterakis spumosa, seguido por los cestodos: Hymenolepis diminuta, H. nana, Moniliformis moniliformis y Cysticercus fasciolaris. Conclusiones. La presencia de cuatro especies de cestodos zoonóticos en los roedores muestreados que viven cerca comunidades humanas, representan un riesgo potencial de infecciónpara los habitantes, especialmente en el caso de $R$. norvegicus (presento mayor diversidad de especies parasitarias).Por lo tanto, el control de la población de roedores en las áreas residenciales y las recomendaciones a la población local sobre el riesgo de transmisión de enfermedades a través de roedores parece ser totalmente necesario.

Palabras clave: Parasitosis; helminto; fauna; cestodos; nemátodo; zoonosis (Fuente: DeCS).

\section{ABSTRACT}

Objective. The present study was to identify gastro-intestinal endoparasitic helminths in wild rats. Materials and methods. a parasitological study was carried out to know the helminth-fauna in different urban and rural areas in five provinces of the Republic of Ecuador, during 2014-2017. The rodents were captured and transported to the National Parasitology Reference Center of the National Institute of Public Health Research - Guayaquil, for further analysis. Results. 125/211 (59.2\%) rats with endoparasites were found, $13 / 20$ (65\%) for $R$. rattus and $112 / 191(58.6 \%) R$. norvegicus. The most prevalent nematode: was Nippostrongylus brasiliensis for both species and Heterakis spumosa; followed by the cestodes: Hymenolepis diminuta, H. nana, Moniliformis moniliformis and Cysticercus

Como citar (Vancouver).

Solórzano AL, Sánchez-Amador F, Sánchez GS, Pizarro VJ. R. rattus y R. norvegicus, como reservorio de endoparásitos zoonóticos en Ecuador. Rev MVZ Córdoba. 2021; 26(3):e1260. https://doi.org/10.21897/rmvz.1260 
fasciolaris. Conclusions. The presence of four species of zoonotic cestodes in the sampled rodents that live near human communities, represent a potential risk of infection for the inhabitants. Therefore, the control of the population of rodents in residential areas and the awareness of the local population about the risk of disease transmission through rodents seems to be totally necessary.

Keywords: Parasitosis; helminth; fauna; cestodes; nematode; zoonoses (Sourece: DeCS).

\section{INTRODUCCIÓN}

El desarrollo económico ha llevado a un aumento de la urbanización, ya que las personas migran de las zonas rurales en busca de oportunidades de empleo y educación (1). En muchos países en desarrollo, esto, a su vez, ha conducido a un aumento de la pobreza urbana, que se refleja en la vivienda deficiente, el apoyo de infraestructura y los servicios sociales y de salud, características que definen los asentamientos de barrios marginales (2). Aunque ha habido iniciativas para reducir la pobreza, como las estrategias emprendidas para alcanzar los Objetivos de Desarrollo del Milenio, el número de residentes de barrios marginales aumentó en un $28 \%$ de 1990 a 2014 (de 689 a 881 millones) $(1,2$ ).

Los roedores son reconocidos como anfitriones de al menos 60 enfermedades zoonóticas que representan una seria amenaza para los humanos $(3,4,5)$. Históricamente, los roedores asiáticos del género Rattus han sido implicados en la emergencia ypropagación de enfermedades infecciosas como la peste, el tifus murino, el tifus, la leptospirosis y la fiebre hemorrágica por hantavirus, entre otros (6). Pueden causar considerable pérdida económica $(7,8)$ y tienen gran impacto en la biodiversidad $(9,10)$.

Estudios en Serbia reportaron en con una infección intestinal por helmintos del $68.5 \%$ de las 302 ratas. Se recuperaron siete especies de helmintos, de las cuales cinco fueron nematodos: H. spumosa, N. brasiliensis, Capillaria sp., Trichuris muns y Syphacia muris, y dos especies de cestodos: $H$. diminuta y Rodentolepis fraterna. Los parásitos más prevalentes fueron H. spumosa (36.7\%) y H. diminuta $(30.5 \%)$ para Rattus spp. (11).

En Latinoamérica, estudios realizados en Medellín, Colombia, se capturaron 254 ejemplares de $R$. norvegicus. Donde se encontraron cisticercos de $T$. taeniaeformis en los hígados de 6 ejemplares que presentaron larvas, con una frecuencia de $2.4 \%(6 / 254)(12)$.
Reportaron frecuencias de infección por: $H$. diminuta, Rodentolepis fraterna, Raillietina demerariensis, Aspiculuris tetraptera, T. muris, Gongylonemaneo plasticum y $M$. moniliformis en ratas en zonas urbanas de la ciudad de Lima, Perú (13).

Se carece de información del impacto que causan estos en la transmisión de agentes etiológicos zoonóticos Ecuador continental; tan solo existen registros de la presencia de $R$. rattus y $R$. norvegicus, en poblaciones rurales (recintos) por estudios de brotes de Angiostrongylus cantonensis, en dos provincias, durante 20082011 (14). Los roedores son reconocidos como reservorios de numerosos patógenos virales, bacterianos y endoparásitos helmintos, que pueden infectar al ser humano por contacto directo por las heces (15).

La evaluación de las helmintiasis de roedores en diferentes áreas geográficas permitirá establecer el impacto de las enfermedades asociadas a los roedores en la salud humana y del ganado (16).

Los países en vías de desarrollo poseen unas características de pobreza, hacinamiento y falta de servicios básicos que permiten el desarrollo de gran cantidad de roedores que conviven con la población, lo que representa un riesgo mayor para la adquisición de enfermedades zoonóticas.

El objetivo del presente estudio fue identificar los helmintos intestinales en ratas silvestres ( $R$. rattus y $R$. norvegicus), en cinco provincias de diferentes regiones para evaluar la distribución de especies de parásitos presentes, y la pertinencia de éstos como reservorioszoonóticos de importancia en salud pública.

\section{MATERIALES Y MÉTODOS}

Capturas y sitio de estudio.Se capturaron 211 roedores durante enero del 2014 y diciembre del 2017, en cinco provincias (Guayas, Santa Elena, Los Ríos, Napo y Cañar), a conveniencia de un proyectode investigación, aprobado 
en el 2013 por el comité científico, de uso de instalaciones, manejo de reactivos y de los animales empleados (supervisado por el responsable del Bioterio), sobre hospederos definitivos de $A$. cantonensis en zonas endémicas del Ecuador. Se decidió entonces registrar la presencia de otras parasitosis gastrointestinales.

Trabajo de campo. Los sitios fueron elegidos estratégicamente, de acuerdo a la literatura citada (14) y se incluyeron lugares nuevos: Guayas (cantones: Guayaquil y Duran), Los Ríos (cantón Babahoyo), Cañar (cantón La Troncal), Napo (cantón Tena) y Santa Elena (recinto Cadeate). Se utilizaron nueve trampas Tomahawk $(15 \times 15 \times 45 \mathrm{~cm})$ de captura viva para ratas con ayuda de cebos no tóxicos (pan, embutidos, pollo yqueso). Se registró: la procedencia y los parámetros físicos (longitud total, largo de cola, patas y orejas y el peso) para establecer la especie $(17,18)$.

Recolección y procesamiento de muestras. Los procedimientos para la captura, traslado y manejo de los animales vivos, fueron acondicionados en las instalaciones dela Plataforma Bioterio del Instituto Nacional de Investigación en Salud Pública "Dr. Leopoldo Izquieta Pérez" de la ciudad de Guayaquil, a una temperatura de $20-25^{\circ} \mathrm{C}$, con una humedad relativa promedio de $40-70 \%$ y un fotoperiodo de luz/oscuridad de $12 / 12$ horas, manteniendo el acceso libre al agua y alimentos. Posteriormente fueron, registradas (numero/origen/sexo) y pesadas.

Los animales fueron manejados de acuerdo con las pautas estipuladas en el Manual sobre el cuidado y Uso de los Animales de Experimentación, Capítulos: II, III, V (Literales: $\mathrm{G}, \mathrm{H})$, IX, X, XI, XII.

Asimismo, se respetaron los derechos universales de los animales de acuerdo con la Declaración de la Asociación Médica Mundial sobre el uso de animales en la investigación Biomédica (19). Tambien, se respetaron los derechos universales de los animales de acuerdo con la Declaración de la Asociación Médica Mundial sobre el uso de animales en la investigación Biomédica (20).

Se examinaron las cavidades abdominales de Rattus spp., en búsqueda de los parásitos: enquistados, estadios larvales, juveniles y adultos, en los diferentes órganos (esófago, hígado, estomago, intestino y colon). Adicionalmente, se observó la presencia de huevos en heces por observación directa en solución salina fisiológica y/o con Lugol. Los huevos debían ser de tipo embrionados o larvados, de cáscara gruesa o delgada, con presencia de ganchos en el escólex, o con presencia o ausencia de filamentos polares, dependiendo de la especie (13).

Para la identificación de helmintos adultos, los nemátodos se clarificaron en una mezcla de alcohol con fenol y los platelmintos se fijaron con formol al $10 \%$. Los detalles morfológicos de los parásitos adultos y huevos fueron observados con ayuda de un microscopio de luz (13). Todos los helmintos fueron procesados usando las técnicas parasitológicas estandarizadas para su identificación al nivel de especies $(13,21)$.

Las frecuencias de presentación de los parásitos se expresan en forma porcentual. Las posibles asociaciones entre las especies de helmintos con las variables especie fueron evaluadas por medio de la prueba de Chi cuadrado y el test exacto de Fisher (13) y el riesgo de infección se determinó mediante razón de prevalencia (RR) evaluado. Los estadísticos se obtuvieron del programa Epi-info 7 (22).

\section{RESULTADOS}

De las doscientas once ratas capturadas, 125 se encontraron parasitadas con algún helminto (59.2\%). R. norvegicus 112/191 (58.6\%) y $R$. rattus $13 / 20(65 \%)$. Se identificaron: $N$. brasiliensis, $H$. spumosa, $H$. diminuta, $H$. nana, $C$. fasciolaris y $M$. moniliformis (Figuras $1,2,3,4,5,6$ ).

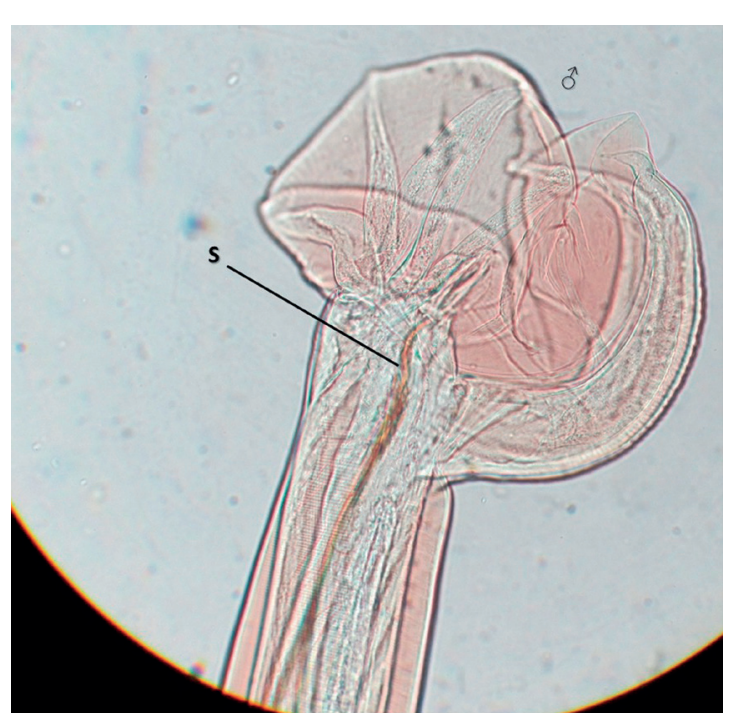

Figura 1. Nippostrongylus brasiliensis adulto mancho, con espículas (s). 


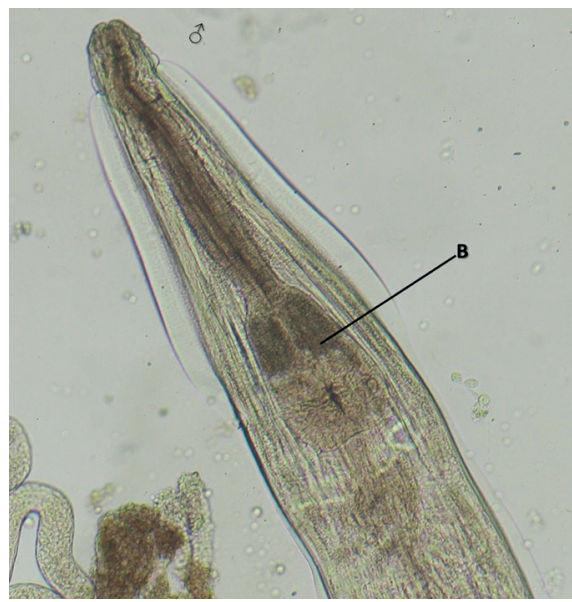

Figura 2. Heterakis spumosa adulto mancho, vista anterior bulbo esofágico (B).

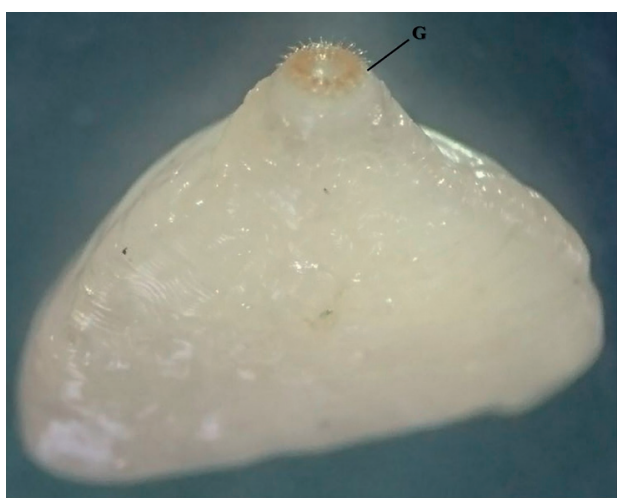

Figura 3. Cisticercus fasciolaris larva L1, corona de ganchos (G) en el escólex.

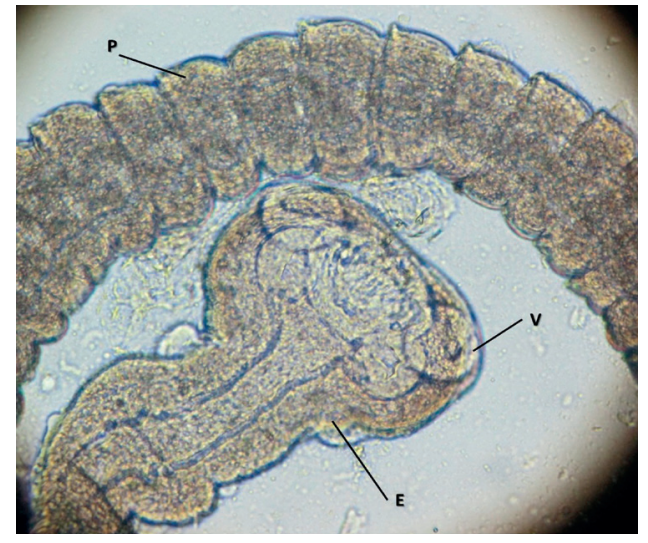

Figura 4. Hymenolepis diminuta adulta, escólex (E) con ventosas $(\mathrm{V})$, y proglotides $(\mathrm{P})$

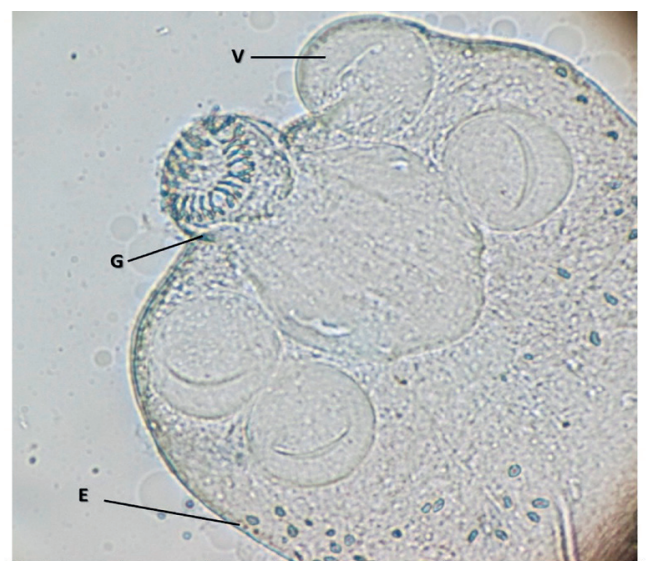

Figura 5. Hymenolepis nana adulta, escólex (E) con ventosas $(V)$ y corona de ganchos $(G)$

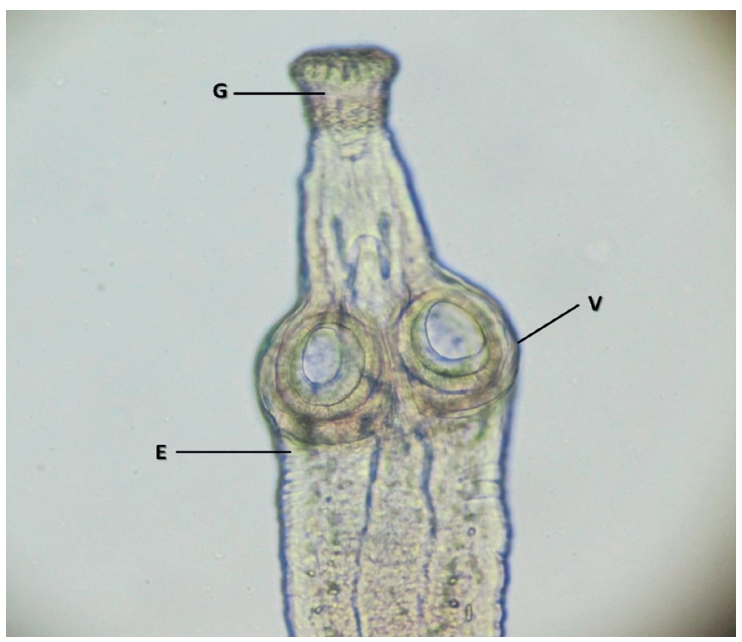

Figura 6. Hymenolepis nana adulta, escólex (E) con ventosas (V) y corona de ganchos (G).

En $R$. rattus se encontraron tres especies de helmintos, un nemátodo, (N.brasiliensis en cinco roedores) y dos cestodos $(H$. diminutaen tres roedores y $C$. fasciolaris en cinco). Para la prueba de Razón de Prevalencia (RR): 1.7 ( $H$. diminuta) y 1.785 y prueba de Fischer: 0.5211 (H. diminuta) y 0.1137 (Tabla 1 ).

Tabla 1. Helmitofauna encontrada en rata negra (Rattus rattus). Ecuador.

\begin{tabular}{ccccccc}
\hline Parásitos & $\begin{array}{c}\text { \# Rattus } \\
\text { Spp }\end{array}$ & $\begin{array}{c}\text { Ratas } \\
\text { infectadas }\end{array}$ & $\begin{array}{c}\text { Prevalencia } \\
\text { Parásitos }\end{array}$ & $\begin{array}{c}\text { Numero } \\
\text { Parásitos }\end{array}$ & $\begin{array}{c}\text { Razón de } \\
\text { prevalencia (RR) }\end{array}$ & p Fisher exact \\
\hline & \multicolumn{7}{c}{ Cestodos } \\
\hline Hymenolepis diminuta & 20 & 3 & $15 \%$ & 11 & 1.7 & 0.5211 \\
Cysticercusfasciolaris. & 20 & 5 & $25 \%$ & 7 & 1.875 & 0.1137 \\
\hline & & \multicolumn{7}{c}{ Nemátodo } & & \\
\hline Nippostrongylusbrasiliensis & 20 & 5 & $25 \%$ & 121 & 1.875 & 0.1137 \\
\hline
\end{tabular}


En $R$. norvegicus se encontraron seis especies de helmintos, dos nemátodos ( $N$. brasiliensisen 112 roedores y $H$. spumosa en tres individuos) y cuatro cestodos $(H$. diminuta en 28 roedores, $H$. nana en 10 roedores, $M$. moniliformis en 5 roedores y $C$. fasciolaris en 21 roedores). Los valores de Razón de Prevalencia: -1, 1.72, $1.94,1.77,1.86,3.62$ respectivamente. Prueba de Fischer:0.26 ( $H$. spumosa), 0.078 ( $M$. moniliformis) y 0.0058 ( $H$. nana), el resto de parásitos con valor cero (Tabla 2).

En la Provincia del Guayas se capturaron 20 $R$. rattus y $165 R$. norvegicus. Mientras que, en Santa Elena, Los Ríos Cañar y Napo se capturaron solamente $R$. norvegicus: $10,3,2$ y 2 respectivamente. En Guayas se capturo y encontró mayor número de parásitos que las demás provincias (Figura 7; Tabla 3).

Adicionalmente, se encontraron seis larvas de Filaria spp., (no identificadas) en el interior del tejido de las arterias coronarias de cuatro $R$. norvegicus.

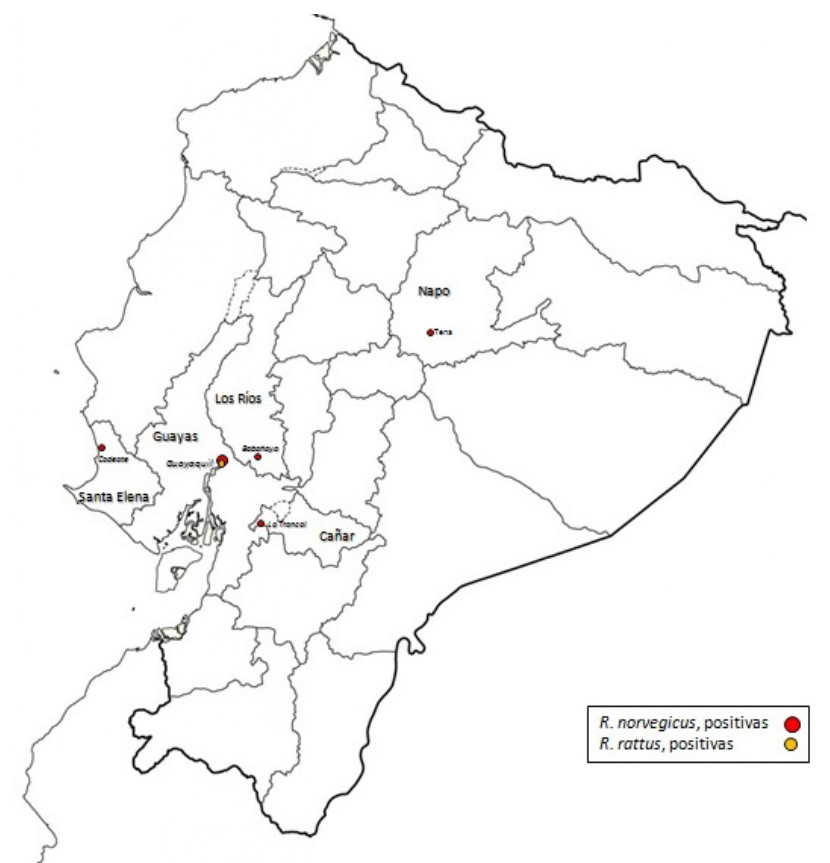

Figura 7. Mapa político del Ecuador con los puntos de referencia donde se capturaron roedores (Rattus spp.) con parásitos gastrointestinales.

Tabla 2. Helmitofauna encontrada en rata parda (Rattus norvegicus) Ecuador.

\begin{tabular}{|c|c|c|c|c|c|c|}
\hline Parásitos & $\begin{array}{l}\text { \# Rattus } \\
\text { spp }\end{array}$ & $\begin{array}{c}\text { Ratas } \\
\text { infectadas }\end{array}$ & $\begin{array}{c}\text { Prevalencia } \\
\text { Parásitos }\end{array}$ & $\begin{array}{c}\text { Numero } \\
\text { Parásitos }\end{array}$ & $\begin{array}{c}\text { Razón de } \\
\text { prevalencia (RR) }\end{array}$ & $\begin{array}{c}\text { p Fisher } \\
\text { exact }\end{array}$ \\
\hline \multicolumn{7}{|c|}{ Cestodos } \\
\hline Hymenolepis diminuta & 191 & 28 & $15 \%$ & 135 & 1.94 & 0 \\
\hline Hymenolepis nana & 191 & 10 & $5 \%$ & 125 & 1.774 & 0.0058 \\
\hline Cysticercusfasciolaris. & 191 & 21 & $11 \%$ & 53 & 1.868 & 0 \\
\hline Monofilformismoniliformis & 191 & 5 & $3 \%$ & 6 & 3.621 & 0.078 \\
\hline \multicolumn{7}{|c|}{ Nemátodo } \\
\hline Nippostrongylusbrasiliensis & 191 & 112 & $59 \%$ & 2519 & -1 & 0 \\
\hline Heterakisspumosa & 191 & 3 & $2 \%$ & 106 & 1.7248 & 0.2686 \\
\hline
\end{tabular}

Tabla 3. Helmitofauna Rattus spp., según las provincias seleccionadas Ecuador.

\begin{tabular}{ccccccc}
\hline $\begin{array}{c}\text { Provincias/ } \\
\text { Parásitos }\end{array}$ & $\begin{array}{c}\text { Hymenolepis } \\
\text { diminuta }\end{array}$ & $\begin{array}{c}\text { Hymenolepis } \\
\text { nana }\end{array}$ & $\begin{array}{c}\text { Cisticercus } \\
\text { Fasciolaris. }\end{array}$ & $\begin{array}{c}\text { Monoliformis } \\
\text { moniliformis }\end{array}$ & $\begin{array}{c}\text { Nippostrongylus } \\
\text { brasiliensis }\end{array}$ & $\begin{array}{c}\text { Heterakis } \\
\text { spumosa }\end{array}$ \\
\hline Guayas & 27 & 10 & 39 & 10 & 73 & 3 \\
Santa Elena & - & - & - & - & 2 & - \\
Los Ríos & - & - & - & - & 1 & - \\
La Troncal & 1 & - & - & - & 5 & - \\
Napo & - & - & - & - & 2 & - \\
Total & 28 & 10 & 39 & 10 & 83 & 3
\end{tabular}




\section{DISCUSIÓN}

En un estudio realizado entre 2009-2013 en diferentes regiones de Holanda, se capturaron 40 $R$. rattus y $117 R$. norvegicus, con nueve especies deendoparásitos, entre ellos: $N$. brasiliensis, $H$. spumosa, $H$. diminuta y $H$. nana (23).

Entre 2010-2011 en São Gonçalo, Rio de Janeiro, Brasil se atraparon $114 R$. norvegicus, solo dos ratas estuvieron libres de infección. Se hallaron un total 14.401 de helmintos en el intestino delgado y grueso. Alrededor del $98.7 \%$ fueron nemátodos, incluyendo $N$. brasiliensis (11.518) y $H$. spumosa (189) y dos especies de cestodos: $H$. nana y $M$. moniliformis (24).

En nuestro estudio de 211 ratas se encontraron 124 parasitadas con algún helminto. $R$. norvegicus $112 / 191$ (58.6) y $R$. rattus $13 / 20$ (65\%), valores inferiores encontrados en otros países.

Aunque las especies de helmintos que se encuentran en $R$. norvegicus son similares en todo el mundo, la estructura de la comunidad de helmintos difiere según las características regionales (por ejemplo, temperatura del aire, $\mathrm{pH}$ del suelo, precipitación y presencia del huésped intermedio). Además, estudios previos han demostrado que los nemátodos y cestodos son más abundantes en las comunidades de $R$. norvegicus (24).

El helminto más prevalente fue $N$. brasiliensis ( $25 \%$ para $R$. rattus y $53 \% R$. norvegicus) y abundante (2519 organismos) de este estudio. Este nemátodo ya ha sido reportado antes en Rattus spp., en otros países en Sudamérica, debido a que adquiere los parásitos por ingestión oral y penetración en la piel de larvas infecciosas (L3), que es el medio más común (24).

Estudios realizados en Brasil han demostrado que, cuando las ratas jóvenes están expuestas a la infección por $N$. brasiliensis, el helminto persiste hasta la vida adulta y las ratas jóvenes no provocan la expulsión del parásito, mientras que las ratas adultas expuestas a $N$. brasiliensis desarrollan una respuesta inmune. Basado en este hecho, la infección de $R$. norvegicus por $N$. brasiliensis observada en el presente estudio puede haber ocurrido durante la edad juvenil, lo que favorece un aumento en la carga parasitaria a través del tiempo. La transmisión también puede estar relacionada con el tamaño del grupo de hospedadores, el tamaño del campo de origen del hospedador y el grado de sociabilidad del hospedero (24).
Hymenolepis spp., fue el segundo helminto más prevalente en este estudio $(13.3 \% \mathrm{H}$. diminuta y $4 \% H$. nana en $R$. norvegicus, y $15 \%$ de $H$. diminuta en $R$. rattus). Estudios anteriores de cestodoshan reportado mayor cantidad Tanto $H$. nana y $H$. diminutason helmintos cestodos zoonóticos, aunque este último se ve con poca frecuencia en los seres humanos $(13,23,24)$. $H$. diminuta se transmite a los humanos por la ingestión de Tribolium confusum (escarabajo de la harina como huésped intermediario) con cereales infestados, o por vía fecal-oral. $H$. nana se transmite a través del contacto fecaloral (huevos), o por la ingestión accidental de hospederos intermediarios (artrópodos) que albergan cisticercoides (25). Las infecciones en los seres humanos por estas dos especies son en su mayoría asintomáticas, aunque pueden producir debilidad, dolor de cabeza, dolor abdominal y diarrea $(23,25)$.

Una especie de helmintos transmitidos por la ingestión de huevos en el suelo es $H$. spumosa (sólo el $1.5 \%$ de $R$. norvegicus infectado). Estudios similares en Sudamérica reportan en ratas (Rattus spp.) altas tasas de infección $(13,23,26)$ que pueden adquirirse en las madrigueras subterráneas, que albergan el parásito (26).

M. moniliformis, con una prevalencia menor que en otros estudios (2.4\%), es un parásito de interés para la salud pública debido a que hay reportes de infecciónen humanos al ingerir el huésped intermedio infectado, que son artrópodos como la cucaracha (24), causando alteraciones gastrointestinales, especialmente en niños, que viven en condiciones de higiene inadecuada, sin instalaciones sanitarias $(24,26)$.

Otro helminto presente en el estudio fue $C$. fasciolaris ( $9.9 \%$ en $R$. norvegicus) menor a otros estudios realizados en Corea del Sur, donde se encontraron en 287 ratas, 97 (33.8\%) infectadas con este parásito (27), corresponde al estado larvario del cestodo $T$. taeniaeformis. Los huevos liberados de las proglótides maduran, salen con las heces y cuando son ingeridos por un animal susceptible, se activan durante el paso por el estómago y las larvas (oncosferas) migran hacia el hígado, donde continúan su desarrollo dentro de un quiste (28). Este helminto ha sido documentado en casos humanos en estudios previos (29). 
El parásito zoonótico más comúnmente reconocido en el presente estudio fue $H$. diminuta, con la mayor tasa de infestación en $R$. norvegicus seguida de $H$. nana, $C$. fasciolaris y M. monoliformis. En estos roedores reviste gran importancia ya constituyen un reservorio permanente para la trasmisión de enfermedades a humanos.

Estudios en Lima Perú mostraronuna asociación estadística entre la infección con este parásito (H. diminuta) y la categoría de las ratas $(R$. rattus mayor y menor a $20 \mathrm{~cm}$ ) con un valor de $p=0.021$, concluyendo que no hay una asociación estadística entre algún otrohelminto de interés zoonótico y las variablesen estudio este estudio (13). De igual forma en nuestro estudio se encontró una asociación entre la infección $(H$. diminuta) para $R$. rattus, con un valor $\mathrm{p}: 0.52$ y para $R$. norvegicus (con $M$. moniliformis) con p:0.078. Debido al que el numero es menor que en relación al estudio antes mencionado.

El hacinamiento y las malas prácticas sanitarias en las zonas urbanas exponen a los habitantes al riesgo de infección. Aunque, en este estudio, las especies de importancia médica fueron menos abundantes, señalamos la necesidad de un control continuo de las poblaciones de ratas, nuevas investigaciones de la ecología de las enfermedades y programas de educación sanitaria para reducir la transmisión humana de parásitos intestinales (26).
En conclusión se han demostrado diferencias considerables en la variación de las especies de helmintos entre: $R$. rattus y $R$. norvegicus. La variación de especies de helmintos encontrada en el intestino (cuatro especies) y las infecciones parasitarias en otros órganos (hígados y arterias coronarias) se registraron más en las ratas pardas que en las ratas negras, las primeras, en el mismo periodo,tuvieronel mayor número de infecciones simultáneas, en comparación con las ratas negras del mismo entorno.

Finalmente los roedores estudiados albergaban varios parásitoszoonóticos, se recomienda la toma de medidas para controlar la población de roedores y la advertencia la población local sobre el riesgo de transmisión de enfermedades a través de roedores.

\section{Conflicto de interés}

Ninguno declarado.

\section{Agradecimiento}

A todos los integrantes del Centro de Referencia Nacional de Parasitología del Instituto Nacional de Investigación en Salud Pública (INSPI) "Leopoldo Izquieta Pérez". Guayaquil-Ecuador. A la Dra. María Beltrán, Manuel Tantaleán y miembros del Departamento de Parasitología del Instituto Nacional de Salud de Perú. Y Sra. Elsa P. Amador Palma por el método de captura empleado.

\section{REFERENCIAS}

1. UN-Habitat. Urbanization and Development: Emerging Features. United Nations Human Settlements Programme: Nairobi, Kenya; 2016. https://unhabitat.org/sites/default/ files/download-manager-files/WCR-2016WEB.pdf

2. Carvalho-Pereira T, Souza F, Santos L, Walker R, Pertile A, Oliveira $D$, et al. The helminth community of a population of Rattus norvegicus from an urban Brazilian slum and the threat of zoonotic diseases. Parasitol. 2018; 145(6):797-806. http:// dx.doi.org/10.1017/S0031182017001755
3. Meerburg BG, Singleton GR, Kijlstra A. Rodent-borne diseases and their risks for public health. Crit Rev Microbiol.2009; 35:221-270. https://doi. org/10.1080/10408410902989837

4. Luis AD, Hayman DT, O'Shea TJ et al. A comparison of bats and rodents as reservoirs of zoonotic viruses: Are bats special? Proc Biol Sci. 2013; 280:20122753. http:// dx.doi.org/10.1098/rspb.2012.2753 
5. Chaisiri K, Siribat $P$, Ribas A, Morand S. Potentially zoonotic helminthiases of murid rodents fromthe Indo-Chinese peninsula: Impact of habitat and therisk of human infection. Vector-Borne Zoonotic Dises. 2015; 15:73-85. https://doi.org/10.1089/ vbz.2014.1619

6. Kosoy M, Khlyap L, Cosson J-F, Morand S. Aboriginal and invasive rats of genus Rattus as hosts ofinfectious agents. Vector-Borne Zoonotic Dises. 2015; 15:3-12. https://doi. org/10.1089/vbz.2014.1629

7. Singleton $G R$, Belmain $S$, Brown $P R$, Aplin K, Htwe NM. Impacts of rodent outbreaks on food security in Asia. Wildlife Research. 2010; 37:355-359. https://doi. org/10.1071/WR10084

8. John A. Rodent outbreaks and rice preharvestlosses in Southeast Asia. Food Security. 2014; 6:249-260. https://doi. org/10.1007/s12571-014-0338-4

9. Wyatt KB, Campos PF, Gilbert MTP et al. Historical mammal extinction on Christmas island (Indian Ocean) correlates with introduced infectious disease. PLOS ONE. 2008; 3:e3602. https://doi.org/10.1371/ journal.pone.0003602

10. Morand S, Bordes F, Chen HW, Claude J, Cosson JF, et al. Global parasite and Rattus rodent invasions: The consequences for rodent-borne diseases. Integ Zoo. 2015; 10:409-423. http://dx.doi. org/10.1111/1749-4877.12143

11. Kataranovski M, Mirkov I, Belij S, Popov A, Petrović Z, Gačić Z, Kataranovski D. Intestinal helminths infection of rats (Rattus norvegicus) In the Belgrade area (Serbia): the effect of sex, age and habitat. Parasite. 2011; 18:189-196. http://dx.doi. org/10.1051/parasite/2011182189

12. Duque $B$, Aranzazu $D$, Agudelo-Flórez P, Londoño A, Quiroz V, Rodas J. Rattus norvegicus como indicador de la circulación de Capillaria hepatica y Taenia taeniaeformis en la Plaza Minorista de Medellín, Colombia. Biomédica. 2012; 32:510-518. http:// dx.doi.org/10.7705/biomedica.v32i4.442
13. Renzo de Sotomayor C, Serrano-Martínez E, Tantaleán M, Quispe HM, Casas VG. Identificación de Parásitos Gastrointestinales en Ratas de Lima Metropolitana. Rev Inv Pe. $2015 ; 26(2): 273-281$. https://doi. org/10.15381/rivep.v26i2.11003

14. Martini RL, Dorta CA. Angiostrongylus cantonensis - Emergencia en América. 1th ed. Editorial Academia: La Habana; 2016. https://www.researchgate.net/ publication/312031778 Angiostrongylus cantonensis Emergencia en America

15. OPS. Manual para el control integral de Roedores. Organización Panamericana de la Salud: Colombia; 2012. https:// www.minsalud.gov.co/sites/rid/Lists/ BibliotecaDigital/RIDE/VS/PP/SA/manualintegral-de-roedores.pdf

16. Ranjbar MJ, Sarkari B, Mowlavi GR, Seifollahi Z, Moshfe A, Abdolahi KS, Mobedi I. Helminth infections of rodents and their zoonotic importance in boyer-ahmad district, southwestern iran. Ira J Parasit. 2017; 12(4):572-579. https://ijpa.tums. ac.ir/index.php/ijpa/article/view/1931

17. Abad AD, Chávez VA, Pinedo VR, Tantaleán VM, Gonzáles-Viera O. Helmintofauna Gastrointestinal de Importancia Zoonótica y sus Aspectos Patológicos en Roedores (Rattus spp) en Tres Medioambientes. Rev Invst Vet Pe. 2016; 27(4):736-750. http:// dx.doi.org/10.15381/rivep.v27i4.12568

18. Milazzo C, Cagnin M, Di BC, Geraci F, Ribas A. Helminth Fauna of Commensal Rodents, Mus musculus (Linnaeus, 1758) and Rattus rattus(Linnaeus, 1758) (Rodentia, Muridae) in Sicily (Italy). Rev IberoLatinoam Parasitol. 2010; 69(2):194-198.

19. CCAC. Guide to the Care and Use of Experimental Animals. 2 Edition. Canadian Council Animal Care: Ottawa, Ontario; 2017. https://www.ccac.ca/Documents/ Standards/Guidelines/Experimental Animals Vol1.pdf

20. AMM. Declaración de la AMM sobre el Uso de Animales en la Investigación Biomédica. Asociación Médica Mundial; 2016. https:// www.wma.net/es/policies-post/declaracionde-la-amm-sobre-el-uso-de-animales-enla-investigacion-biomedica/ 
21. Anderson RC, Chabaud AG, Willmott S. Keys to the nematode parasites of vertebrates. Archivalvolume. Wallingford, UK: CAB International; 2009. https://www.cabi.org/ bookshop/book/9781845935726/

22. CDC. EpiInfo TM. Centers Disease Control; 2017. https://www.cdc.gov/epiinfo/index. $\underline{\text { html }}$

23. Franssen F, Swart A, Knapen F, Giessen J. Helminth parasites in black rats (Rattus rattus) and brown rats (Rattus norvegicus) from different environments in the Netherlands. Infect Ecol Epidemiol. 2016; 6:314-333. http://dx.doi.org/10.3402/iee. v6.31413

24. Simões OR, Luque JL, Gentile R, Rosa MCS, Costa-Neto S, Maldonado A. Biotic and abiotic effects on the intestinal helminth community of the brown rat Rattus norvegicus from Rio de Janeiro, Brazil. J Helminthol. 2014; 90(1):21-27. http://dx.doi.org/10.3402/ iee.v6.3141310.1017/S0022149X14000704

25. Coomansingh $C$, Pinckney RD, Bahaiyat MI, Chikweto A, Bitner S, Baffa A, Sharma $R$. Prevalence of endoparasites wild rats in Grenada. St. George's University. West Ind Vet J. 2009; 9(1):17-21. https://sta.uwi. edu/fms/vet/documents/3.pdf
26. Akande AO. A study on wild rat behaviour and control on a pig farm. Master of Science Programme in Veterinary Medicine for International Students. Swedish: University of Agricultural Sciences; 2011. https://stud. epsilon.slu.se/3357/

27. Gonçalves $A L$, Belizário $T L$, Pimentel JB, Penatti MP, Pedroso RS. Prevalence of intestinal parasites in preschool children in the region of Uberlândia, State of Minas Gerais, Brazil. Rev Soc Bras Med Trop. 2011; 44(2):191-193. https://doi.org/10.1590/ S0037-86822011005000022

28. Al-Salihi KA, Sheikh A, Saied H. Highly prevalence of Strobilocercus fasciolaris infection associated with gastroentropathy and hepatic fibrosarcoma between laboratory rats in experimental gropus. Afri J Anim Biomed Sci. 2009; 4(2):6-10. https://www. ajol.info/index.php/ajbr/issue/archive/2

29. Rodríguez-Vivas R, Panti-May J, Parada-López J, Hernández-Betancourt S, Ruiz-Piña $H$. The occurrence of the larval cestode Cysticercus fasciolaris in rodent populations from the Cuxtal ecological reserve, Yucatan, Mexico. J Helminthol. 2011; 85(4):458-461. http:// dx.doi.org/10.1017/S0022149X10000817 